\title{
Patología unilateral de senos paranasales con compromiso predominante de un seno. Descripción de serie de casos
}

\section{Unilateral paranasal sinus disease with predominant commitment of one sinus. Case series description}

\author{
Karin Krauss $\mathbf{M}^{1}$, Rodolfo Nazar $\mathbf{S}^{1}$, Alfredo Naser $\mathbf{G}^{\mathbf{1}}$, Natalia Cabrera $\mathbf{S}^{1}$.
}

\begin{abstract}
RESUMEN
Introducción: El velamiento de una cavidad paranasal representa un desafío diagnóstico y puede ser causado por diversas patologías. El examen clínico generalmente es insuficiente para orientar la conducta siendo necesario un estudio completo que incluya endoscopía, imágenes e histología para lograr un diagnóstico definitivo.

objetivo: Describir la impresión clínica, imagenológica, quirúrgica e histológica de pacientes con patología unilateral de senos paranasales.

Material y método: Serie retrospectiva de 32 pacientes con patología sinusal unilateral operados en Hospital Clínico de la Universidad de Chile entre 2012 y 2014. Se consideró como criterio de inclusión el compromiso predominante de un seno paranasal en el estudio imagenológico. Se describen las variables: síntoma principal, impresión diagnóstica; clínica, imagenológica, quirúrgica, seno comprometido, entre otras.

Resultados: El síntoma principal más frecuente fue obstrucción nasal (56\%). Los diagnósticos más frecuentes fueron: patología inflamatoria (84\%): pólipo antrocoanal (31\%), mucocele (22\%) y bola fúngica (22\%) y patología tumoral benigna: papiloma invertido (16\%), siendo el seno maxilar el más afectado (90\%). La impresión diagnóstica quirúrgica fue más precisa que la imagenológica en determinar el diagnóstico definitivo, aunque esta diferencia no alcanzó significancia estadística.
\end{abstract}

Discusión: Patología compleja que requiere estudio completo incluyendo endoscopía. Las imágenes son el segundo pilar en el diagnóstico y deben solicitarse de acuerdo a los hallazgos. El diagnóstico quirúrgico provee una mayor precisión diagnóstica.

Conclusiones: Se incluyen varias patologías bajo este concepto, la patología inflamatoria predomina pero se debe tener alto índice de sospecha dado la relativa frecuencia de patología tumoral.

Palabras clave: Sinusitis unilateral, velamiento unilateral, síntomas nasosinusales unilaterales, masa nasal unilateral.

1 Médico Otorrinolaringólogo. Hospital Clínico Universidad de Chile. 


\section{ABSTRACT}

Introduction: Paranasal sinus opacity represents a diagnostic challenge and can be caused by diverse pathologies. Clinical examination is generally insufficient to achieve a definitive diagnosis and it is necessary a complete study including endoscopy, imaging and histology.

Aim: To describe clinical, radiographical, surgical and histological diagnostic impressions of patients with unilateral sinus disease.

Material and method: 32 retrospective serie with unilateral sinus disease treated at Hospital Clínico de la Universidad de Chile, between 2012 and 2014. The predominant commitment of one sinus on the tomography was considered as inclusion criterion. Principal symtom, clinical, radiographical and surgical diagnostic impressions, committed sinus, among others variables were studied.

Results: The most frequent symtom was nasal obstruction (56\%). The most frequent diagnoses were: inflammatory disease (84\%): antrochoanal polyp (31\%), mucocele (22\%) and fungus ball (22\%) and benign tumor: inverted papilloma (16\%). The maxillary sinus was affected in 90\%. Surgical diagnostic impression was more precise than radiographical impression in determining the definitive diagnosis, although this difference did not reach statistics significance.

Discussion: Unilateral sinus disease is a complex pathology that requires comprehensive study including endoscopy. Images are the second pillar in the diagnosis and should be requested according to the findings. Surgical diagnostic impression provides greater diagnostic presicion.

Conclusions: Several pathologies are included under this concept, inflammatory disease prevails but it is necessary to have high index of suspicion in view of the relative frequency of tumour pathology.

Key words: Unilateral sinus disease, sinonasal opacity, unilateral sinonasal symptoms, unilateral nasal mass.

\section{INTRODUCCIÓN}

La patología unilateral de las cavidades paranasales (CPN) representa un desafío diagnóstico debido a la diversidad de patologías que pueden estar implicadas y por tanto la variedad de presentaciones clínicas posibles. En ocasiones puede ser pesquisada como hallazgo en los estudios de imágenes y en otras puede provocar sintomatología como obstrucción nasal, dolor facial, rinorrea o epistaxis y cuando estos síntomas son unilaterales deben alertar al tratante para realizar un estudio acucioso. Hay autores que incluso consideran que la presencia de un pólipo o una masa unilateral debe ser considerada como una lesión neoplásica, hasta que se demuestre lo contrario ${ }^{1}$. La edad es un factor importante a considerar y como en muchas otras patologías a medida que aumenta la edad hay mayor riesgo de patología maligna².

La diferenciación clínica y radiológica entre las patologías que pueden generar síntomas unilatera- les como la rinosinusitis crónica, papiloma invertido, carcinoma espinocelular, entre otros puede ser muy compleja ${ }^{3}$. Otros diagnósticos que deben ser considerados ante la presencia de opacificación unilateral de CPN son la poliposis nasosinusal, la sinusitis fúngica, los mucoceles y las neoplasias benignas y malignas. Los quistes de retención, granulomas de colesterol y la sinusitis de origen dental son causas menos frecuentes ${ }^{4}$.

En la actualidad se cuenta con diversas alternativas radiológicas para profundizar en el diagnóstico como la tomografía computarizada (TC), la resonancia nuclear magnética (RNM), la angiografía, entre otras. Dado su mayor disponibilidad la TC es la más comúnmente usada, pero en muchas situaciones esta herramienta es incapaz de diferenciar entre tejido inflamatorio y tejido tumoral. Especialmente en las etapas tempranas, cuando aún no hay compromiso óseo de las estructuras adyacentes ${ }^{1}$. Existen escasos signos radiológicos 
específicos para una determinada patología y en la mayoría de los casos estos signos son orientadores pero no exclusivos. Se destaca el compromiso óseo en las patologías tumorales, el compromiso de la pared nasal lateral y del meato medio asociado a una superficie lobulada en el papiloma invertido ${ }^{5}$, la opacificación heterogénea parcial 0 completa de un seno asociado o no a microcalcificaciones y esclerosis ósea en la bola fúngica ${ }^{6} 0$ la presencia de calcificación con forma y densidad de pieza dental en el seno maxilar, asociado a no a una fístula oroantral o la presencia de un absceso periapical en las sinusitis de origen dental ${ }^{7}$. En relación a la rinosinusitis crónica estudios han demostrado que los hallazgos imagenológicos de la TC no siempre se correlacionan con los hallazgos quirúrgicos ${ }^{8}$ y en cuanto a la correlación de los hallazgos tomográficos con la histopatología, los escasos estudios existentes no han logrado demostrar una asociación consistente ${ }^{9,10}$.

El diagnóstico definitivo estará determinado entonces, por una valoración íntegra de todas y cada una de las etapas que incluye el proceso: la impresión clínica, imagenológica, quirúrgica e histológica.

\section{OBJETIVOS}

El objetivo del presente estudio es describir la presentación clínica, imagenológica, quirúrgica e histológica de pacientes con patología unilateral de senos paranasales, con compromiso predominante de un seno. Secundariamente se desea describir la concordancia entre la impresión diagnóstica imagenológica y quirúrgica en relación al diagnóstico definitivo.

\section{MATERIAL Y MÉTODO}

Se presenta una serie retrospectiva de 32 pacientes con patología sinusal unilateral operados en el Hospital Clínico de la Universidad de Chile (HCUCH) entre los años 2012 y 2014. Para la selección de los casos se consideró como criterio de inclusión el compromiso predominante de un seno paranasal en el estudio imagenológico. Se describen variables biodemográficas edad y género; y las variables clínicas síntoma principal, impresión diagnóstica clínica, impresión diagnóstica imagenológica, seno comprometido, lado comprometido, impresión diagnóstica quirúrgica, realización de cultivo y su resultado cuando corresponde. Para elaborar el diagnóstico definitivo se consideraron las impresiones diagnósticas clínicas, imagenológicas, quirúrgicas e histológicas. El análisis se realizó con el programa Stata ${ }^{\circledR}$ versión 12. La variable continua (edad) fue descrita como media y rango, y las variables nominales como frecuencias absolutas y relativas. Se utilizó la prueba estadística Ttest para la comparación de promedios de edad y Prtest para evaluar la precisión de la impresión diagnóstica imagenológica y quirúrgica en comparación al diagnóstico definitivo, considerándose un $\alpha$ de $5 \%$. Se contó con la aprobación del Comité de Ética institucional para la realización del presente estudio y el consentimiento informado de cada paciente estudiado.

\section{RESULTADOS}

Se estudió un total de 32 casos. 59\% correspondió a género femenino (19/32), 41\% a género masculino (13/32). La media de edad fue de 41 años ( \pm 22 años) con un rango de 9 a 82. La media edad en mujeres fue 35 años ( \pm 24 años) y en hombres 49 años ( \pm 17 años) no hubo diferencias estadísticamente significativas entre la media de edad entre hombres y mujeres (Ttest with equal variance $p$ : 0.07). La media de edad según diagnóstico se describe en la Tabla 1. El síntoma más frecuente fue obstrucción nasal, 56\% (18/32). (Tabla 2). En relación al examen endoscópico, al 66\% (21/32) se les realizó endoscopía 0 nasofibroscopía. Las impresiones diagnósticas clínicas más frecuentes fueron rinosinusitis crónica no polipoidea (34\%) (11/32), pólipo antrocoanal $(25 \%)(8 / 32)$ y rinitis alérgica $(15 \%)(5 / 32)$ (Tabla 3). En cuanto a la modalidad de estudio inicial en $\mathrm{HCUCH}$, en $91 \%$ se realizó con TC y en 9\% RNM. Las impresiones diagnósticas imagenológicas más frecuentes fueron pólipo antrocoanal $(31 \%)(10 / 32)$ y mucocele $(18 \%)$ (6/32) (Tabla 4).

El seno maxilar fue el más afectado $(90 \%)$ (29/32) (Figuras 1 y 2$)$, seguido de etmoides (6\%) $(2 / 32)$ y esfenoides $(3 \%)(1 / 32)$ (Figura 3). El lado 
Tabla 1. Media de edad según diagnósticos definitivos

\begin{tabular}{|lcc|}
\hline Diagnóstico & $\begin{array}{c}\text { Promedio edad } \\
\text { (años) }\end{array}$ & $\begin{array}{c}\text { Desviación estándar } \\
\text { (años) }\end{array}$ \\
\hline Mucocele & 36 & 11 \\
Polipo antrocoanal & 26 & 21 \\
RSC P & 25 & 19 \\
Bola fúngica & 67 & 7 \\
Papiloma invertido & 45 & 15 \\
Quiste de retención & 17 & 0 \\
\hline
\end{tabular}

RSC P: Rinosinusitis crónica polipoídea.

Tabla 3. Frecuencia absoluta y relativa de impresión diagnóstica clínica

\begin{tabular}{|lcl|}
\hline & Frecuencia absoluta & $\%$ \\
\hline RSC NP & 11 & 34,4 \\
Pólipo antrocoanal & 8 & 25 \\
Rinitis alérgica & 5 & 15,6 \\
Tumor & 4 & 12,5 \\
Mucocele & 2 & 6,3 \\
RSC P & 1 & 3,1 \\
Papiloma Invertido & 1 & 3,1 \\
\hline
\end{tabular}

RSC NP: Rinosinusitis crónica no polipoídea. RSC P: Rinosinusitis crónica polipoídea. 21/32 pacientes fueron evaluados con nasofibroscopía o endoscopía nasal.
Tabla 2. Frecuencia absoluta y relativa síntoma principal

\begin{tabular}{|lcr|}
\hline & Frecuencia absoluta & $\%$ \\
\hline Obstrucción nasal & 18 & 56,3 \\
Cefalea & 4 & 12,5 \\
Descarga posterior & 3 & 9,3 \\
Rinorrea & 2 & 6,3 \\
Exoftalmos & 1 & 3,1 \\
Hipoacusia & 1 & 3,1 \\
Aumento de volumen localizado & 1 & 3,1 \\
Síndrome febril & 1 & 3,1 \\
Epistaxis & 1 & 3,1 \\
\hline
\end{tabular}

Tabla 4. Frecuencia absoluta y relativa de la impresión diagnóstica imagenológica

\begin{tabular}{|lcc|}
\hline & Frecuencia absoluta & $\%$ \\
\hline Pólipo antrocoanal & 10 & 31,3 \\
Mucocele & 6 & 18,7 \\
Bola fúngica & 4 & 12,5 \\
Papiloma invertido & 4 & 12,5 \\
RSC NP & 3 & 9,3 \\
RSC P & 2 & 6,3 \\
Quiste de retención & 2 & 6,3 \\
Cuerpo extraño de tipo dental & 1 & 3,1 \\
\hline
\end{tabular}

RSC NP: Rinosinusitis crónica no polipoídea. RSC P: Rinosinusitis crónica polipoídea.

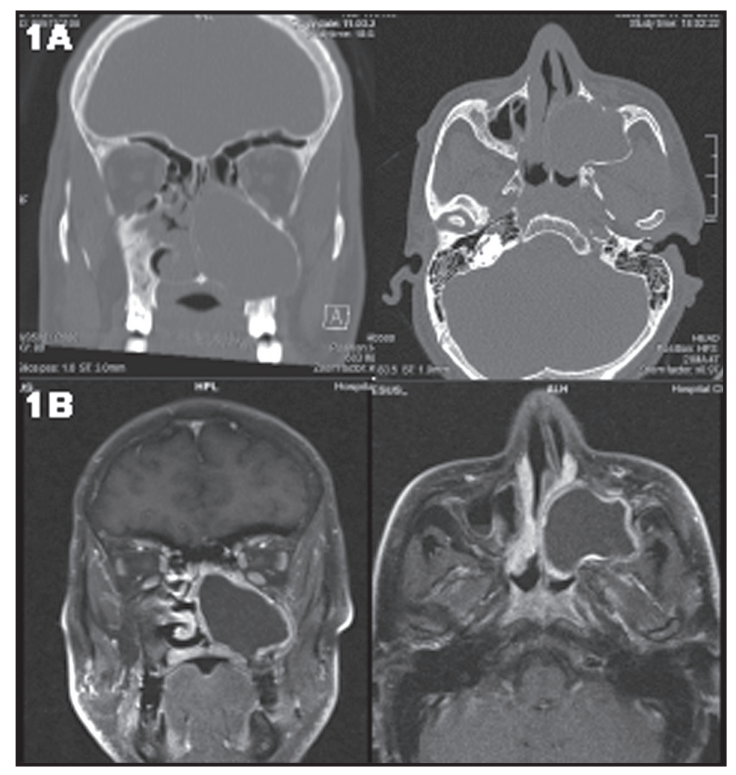

Figura 1. 1A. TC CPN, corte coronal y axial. Lesión seno maxilar izquierdo que compromete la fosa nasal correspondiente a mucocele maxilar. 1B. RNM CPN, corte coronal y axial con la misma lesión con captación intensa periférica e hipointensidad central. 


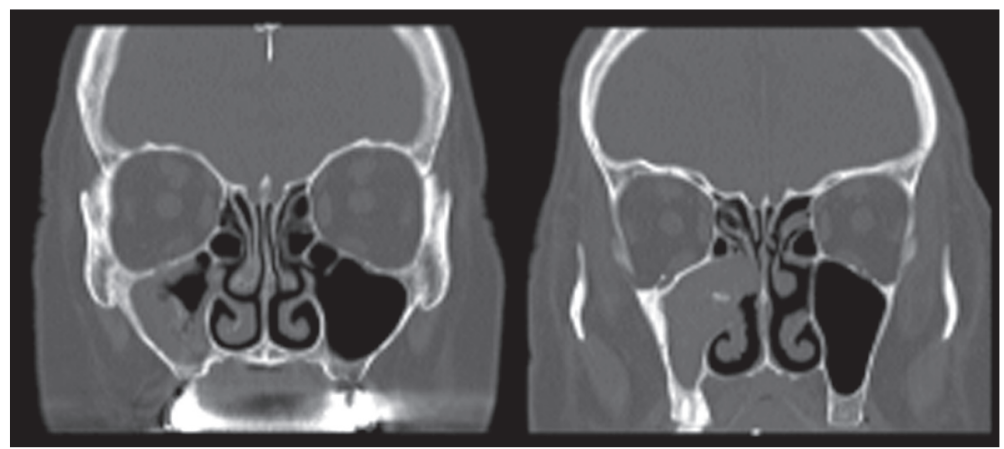

Figura 2. TC CPN, corte coronal. Se evidencia opacificación heterogénea del seno maxilar derecho asociado a esclerosis ósea y calcificaciones correspondiente a bola fúngica.

Figura 3. Imagen endoscópica en intraoperatorio. A derecha (flecha), bola fúngica extraída desde seno esfenoidal. A izquierda ampliación ostium esfenoidal posterior a la extracción de la bola.

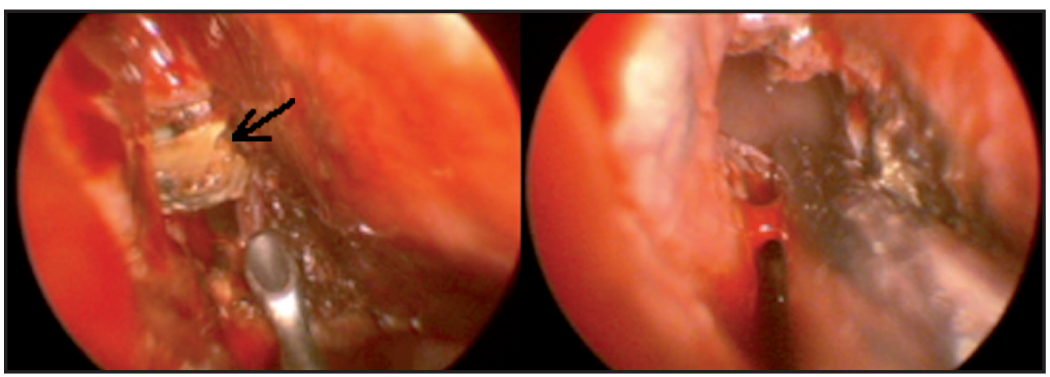

más afectado fue el derecho en el 66\% (21/32). Siendo la diferencia de proporción entre lado derecho e izquierdo estadísticamente significativa (Prtest p: 0.01).

Las frecuencias absolutas y relativas de las impresiones diagnósticas quirúrgicas se describen en la Tabla 5.

Se realizó cultivo en $7 / 32$ pacientes, correspondiendo al $22 \%$. De estos 7 cultivos, 4 resultaron positivos, aislándose los siguientes gérmenes:

Tabla 5. Frecuencia absoluta y relativa de la impresión diagnóstica quirúrgica

\begin{tabular}{|lcr|}
\hline & Frecuencia absoluta & $\%$ \\
\hline Mucocele & 6 & 18,8 \\
Pólipo antrocoanal & 10 & 31,3 \\
RSC NP & 1 & 3,1 \\
RSC P & 2 & 6,3 \\
Bola fúngica & 7 & 21,8 \\
Papiloma invertido & 3 & 9,4 \\
RSC de origen dental & 1 & 3,1 \\
Quiste de retención & 2 & 6,2 \\
\hline
\end{tabular}

RSC NP: Rinosinusitis crónica no polipoídea. RSC P: Rinosinusitis crónica polipoídea.
Staphylococcus aureus, Morganella morganii, Peptostreptococcus sp y Bacilos gram (-).

La impresión diagnóstica quirúrgica fue más precisa coincidiendo en $84 \%$ con el diagnóstico definitivo, por su parte la impresión diagnóstica por imágenes coincidió en $68 \%$, no habiendo diferencia estadísticamente significativa entre estas dos proporciones (Prtest p: 0.14).

En la Tabla 6 se describen los diagnósticos definitivos.

Tabla 6. Frecuencia absoluta y relativa de los diagnósticos definitivos

\begin{tabular}{|lcr|}
\hline & \multicolumn{2}{c|}{ D. Definitivo } \\
& Frecuencia absoluta & $\%$ \\
\hline Patología inflamatoria & 27 & 84 \\
Pólipo antrocoanal & 10 & 31 \\
Mucocele & 7 & 22 \\
Bola fúngica & 7 & 22 \\
RSC P & 1 & 3 \\
RSC de origen dental & 1 & 3 \\
Quiste de retención & 1 & 3 \\
Patología tumoral & 5 & 16 \\
Papiloma invertido & 5 & 16 \\
\hline
\end{tabular}

RSC P: Rinosinusitis crónica polipoídea, RSC: Rinosinusitis crónica. 


\section{DISCUSIÓN}

La patología unilateral de CPN requiere de un estudio acucioso del paciente, una historia clínica completa, un examen físico detallado que incluya endoscopía nasal como pilares primarios del estudio ${ }^{4}$. Aun realizando acabadamente este proceso en muchos casos el examen clínico no será capaz de orientar hacia un diagnóstico en particular, especialmente cuando la patología se encuentra confinada sólo a los márgenes de la CPN sin comprometer la fosa nasal, en estos casos sólo se podrá inferir el lado comprometido pero difícilmente se podrá determinar la CPN afectada. La endoscopía nasal cobra un rol fundamental ya que permitiría observar y detectar hallazgos patológicos tales como secreción purulenta emergiendo desde el meato medio, abombamiento de la pared medial del seno maxilar, presencia de hifas que orienten hacia una infección micótica, degeneración polipoídea del cornete medio o del proceso unciforme, presencia de pólipos o lesiones de aspecto tumoral. A pesar de ellos, en algunos casos hallazgos patológicos específicos pueden no ser detectados por la endoscopía ${ }^{4}$. El estudio con imágenes sigue siendo el segundo pilar fundamental en el proceso diagnóstico, especialmente en aquellos pacientes que se sospeche requerirán de tratamiento quirúrgico posterior. Se hace indispensable para poder determinar la extensión de la enfermedad y el compromiso óseo, para poder determinar él o los senos comprometidos y para realizar la planificación quirúrgica. La mayoría de los autores concuerda que el estudio imagenológico debe iniciarse con TC de CPN ${ }^{1,11,12}$ y en nuestra serie 29/32 (91\%) pacientes fueron estudiados inicialmente con esta herramienta. Cabe mencionar que en los 3 casos que en se optó por realizar inicialmente RNM, correspondían a pacientes que habían sido derivados desde otros centros y contaban con TC previa, por ende finalmente el $100 \%$ de los pacientes contó con estudio tomográfico. Dado que se utilizó como criterio de inclusión el compromiso predominante de un seno paranasal, todos nuestros pacientes presentaban opacificación completa o parcial de al menos una CPN. Es importante recordar que dado la fisiología y anatomía de las CPN es poco probable que exista compromiso aislado de un seno y ya sea por efecto de obstrucción de las vías de drenaje de los senos adyacentes o por mecanismos inflamatorios de la mucosa rinosinusal generalmente se presenta compromiso en otras CPN.

En cuanto al síntoma principal, obstrucción nasal, nuestro resultado se corresponde con el síntoma más frecuente publicado en otras series ${ }^{3,4,13}$.

En relación a los diagnósticos encontrados, la mayor prevalencia fue de patología inflamatoria (pólipo antrocoanal, mucocele, RSC P, bola fúngica, RSC de origen dental y quiste de retención) que en suma representa el $84 \%$ de nuestra serie, este resultado es algo mayor que el de las series publicadas en la literatura internacional, en las cuales la prevalencia de patología inflamatoria va desde $46 \%{ }^{3}$ a $68 \%{ }^{13}$. El $16 \%$ restante de nuestra serie presentó patología tumoral benigna (papiloma invertido) no encontrándose casos de patología tumoral maligna. Esta situación puede deberse a la baja prevalencia de tumores malignos de cavidad nasal y senos paranasales, que corresponde a $3 \%$ de los carcinomas de cabeza y cuello, y sólo al $0,5 \%$ de todas las neoplasias ${ }^{14}$ con una incidencia en nuestro medio de aprox. 0,5-1:100.000 por año $0^{15}$.

Respecto a la media de edad según diagnóstico definitivo, los promedios más altos se concentraron en dos patologías, bola fúngica (media 67 años) y papiloma invertido (media 45 años), resultado que se correlaciona con edad de presentación de dichas patologías 6,16 .

El seno maxilar fue el más afectado y en nuestra serie correspondió al 90\% de los casos, al igual que en la serie reportada por Lee, 2008.

Finalmente en cuanto al grado de concordancia entre la impresión diagnóstica imagenológica y quirúrgica, encontramos una mayor precisión en la impresión diagnóstica quirúrgica (84\%). La ausencia de una diferencia estadísticamente significativa entre proporción de concordancia de ambas modalidades diagnósticas (imagen-cirugía), puede deberse al pequeño tamaño muestral (N:32). La literatura internacional disponible concentra 3 series que incluyen el concepto de patología unilateral de CPN (Ahsan; 2005 en Escocia, Lee; 2008 en Corea del Sur y Habesoglu; 2010 en Turquía) pero en ninguna de ellas se reporta la precisión diagnóstica de las imágenes o la cirugía. La única serie que reporta el porcentaje de diagnóstico 
adecuado tanto de TC, RNM como biopsia incisional en patología unilateral, es una serie japonesa publicada por Ikeda en 1997, que reporta $63 \%$ de concordancia de TC, $64 \%$ de la RNM y $88 \%$ de la biopsia'. Sin embargo es importante mencionar que esta serie consideró como criterio de inclusión la ausencia de destrucción ósea en la TC, por ende gran parte de la patología tumoral, tanto benigna como maligna, no está representada en esta serie. Aun así su porcentaje de diagnóstico adecuado de TC en patología inflamatoria (63\%) es similar a nuestro porcentaje (68\%).

\section{CONCLUSIONES}

Patología unilateral de cavidades perinasales es un concepto que agrupa varias entidades cuyas etiologías pueden ser muy variadas, representa un reto diagnóstico en el cual debe existir un alto índice de sospecha, especialmente considerando que un porcentaje no menor puede corresponder a patología tumoral. El enfrentamiento de estos pacientes debe considerar todas las etapas del proceso diagnóstico; una historia clínica acuciosa, endoscopía o nasofibroscopía nasal, imagenología en forma escalonada dependiendo de los hallazgos tomográficos, estudio histológico y con todos estos antecedentes elaboran un diagnóstico definitivo.

Creemos que dada la complejidad de los diagnósticos que se engloban bajo este concepto, estos pacientes debiesen ser discutidos en un comité especializado, de forma de poder brindar las mejores alternativas terapéuticas y planificar vías de abordaje quirúrgico ajustadas para cada caso particular.

Como todo estudio descriptivo retrospectivo presenta sesgos, particularmente en relación al registro incompleto de los datos en las fichas clínicas, pudiese ser que el porcentaje real de estudio endoscópico realizado a estos pacientes haya sido mayor, pero al no quedar registrado el procedimiento, se consideró como no realizado. Por otro lado el Hospital Clínico de la Universidad de Chile es una institución privada, pudiese existir sesgo de selección de los casos y además algunas patologías podrían verse menos representadas debido al menor acceso al hospital de población de nivel socioeconómico más bajo.
Finalmente consideramos que se requiere de un mayor número de casos para obtener conclusiones más robustas en relación a la concordancia de las impresiones diagnósticas, pero a nivel local sienta las bases para nuevas líneas de investigación.

Los autores declaran no tener conflictos de interés.

\section{BIBLIOGRAFÍA}

1. Ikeda K, Tanno N, Suzuki H, Oshima T, Kano S TT. Unilateral Sinonasal Disease Without Bone Destruction. Arch Otolaryngol Head Neck Surg 1997; 123: 198-200.

2. Lehnerdt G, Weber J, Dost P. [Unilateral opacification of the paranasal sinuses in CT or MRI: an indication of an uncommon histological finding]. Laryngorhinootologie 2001; 80(3): 141-5.

3. Ahsan F, El-Hakim H, Ah-See KW. Unilateral opacification of paranasal sinus CT scans. Otolaryngol - Head Neck Surg 2005; 133(2): 178-80.

4. LEE JY. Unilateral paranasal sinus diseases: analysis of the clinical characteristics, diagnosis, pathology, and computed tomography findings. Acta Otolaryngol 2008; 128(6): 621-6.

5. Dammann F, Pereira P, Laniado M, Plinkert P, LöWENHEIM H, CLAussen CD. Inverted papilloma of the nasal cavity and the paranasal sinuses: using CT for primary diagnosis and follow-up. AJR Am J Roentgenol 1999; 172(2): 543-8.

6. Grosjean P, Weber R. Fungus balls of the paranasal sinuses: A review. Eur Arch Oto-RhinoLaryngology 2007; 264(5): 461-70.

7. Regimantas Simuntis, Ricardas Kubilius SV. Odontogenic maxillary sinusitis: A review. Stomatol Balt Dent Maxillofac J 2014; 16(2): 39-43.

8. JiANETTO D PM. Correlation between preoperative computed tomography and operative findings in functional endoscopic sinus surgery. Laryngoscope 1995; 105: 924-7.

9. GoldWyn BG, SaKR W, Marks SC. Histopathologic Analysis of Chronic Sinusitis. Am J Rhinol 1995; 9(1): 27-30. 
10. Cousin JN, HAR-EL G, LI J. Is there a correlation between radiographic and histologic findings in chronic sinusitis? J Otolaryngol 2000; 29(3): 170-3.

11. Mason JD, Jones NS, Hughes RJ, Holland IM. A systematic approach to the interpretation of computed tomography scans prior to endoscopic sinus surgery. J Laryngol Otol 1998; 112(10): 986-90.

12. JONES NS. CT of the paranasal sinuses: A review of the correlation with clinical, surgical and histopathological findings. Clin Otolaryngol Allied Sci 2002; 27(1): 11-7.
13. Habesoglu TE, Habesoglu M, Surmeli M, Uresin T, EGELI E. Unilateral sinonasal symptoms. J Craniofac Surg 2010; 21(6): 2019-22.

14. Roush GC. Epidemiology of cancer of the nose and paranasal sinuses: current concepts. Head Neck Surg 1979; 2(1): 3-11.

15. Quitral R, Rahal M, Morales I, Daszenies C, Vallejos MP. Tumores malignos de nariz y cavidades paranasales. Revisión de 11 años. Rev Otorrinolaringol Cir Cabeza Cuello 2003; 63: 21-8.

16. Eggers G, Mühling J, Hassfeld S. Inverted papilloma of paranasal sinuses. J CranioMaxillofacial Surg 2007; 35(1): 21-9. 